\title{
Some Morphological Operators on Simplicial Complex Spaces
}

\author{
Fábio Dias, Jean Cousty, and Laurent Najman \\ Université Paris-Est, Laboratoire d'Informatique Gaspard-Monge, \\ Équipe A3SI, ESIEE \\ $\{$ diasf,$j$.cousty, 1. najman\}@esiee.fr
}

\begin{abstract}
In this work, we propose a framework that allows to build morphological operators for processing and filtering objects defined on (abstract) simplicial complex spaces. We illustrate with applications to mesh and image processing, for which, on the provided examples, the proposed approach outperforms the classical one.
\end{abstract}

\section{Introduction}

Introduced by Poincaré [11] for studying the topology of spaces of arbitrary dimensions, a simplicial complex can be seen as a mesh, i.e. a space with a triangulation. The basic building block of the complex is the cell, which can be thought of as a set of elements having various dimensions glued together according to certain rules (e.g., a triangle, its edges and vertices). Although simplicial complexes have a wide variety of different usages (e.g., in computer graphics, in Computer-Aided Design or in modeling), their processing has mostly been considered in term of simplification, for example to obtain a simpler model with less details. However, it is more and more frequent to have data associated with the elements of a mesh (e.g. a curvature or a texture). Processing (filtering) the values associated with a mesh is not a common problem per se in the literature. On the other hand, filtering is a common theme in image processing, and abstract (simplicial or cubical) complexes have been promoted, in particular by Kovalevsky [7, in order to provide a sound topological basis for image analysis, and are more and more popular $41 / 2$. Then again, the values are most of the time located on one of the elements of the cell, usually the facet, i.e. the largest element of the cell. In a purely discrete perspective, being able to deal with smaller elements of the cell will allow a kind of "subpixelic" processing.

In this perspective, mathematical morphology provides a useful toolbox made of non-linear operators. Thanks to their algebraic definitions in the framework of lattices, those morphological operators can be applied to many kinds of organized information, and in particular to simplicial complexes.

The complexes can be considered as a natural generalization of the graphs in the sense that a (symmetric) graph is a one dimensional complex. In the past, several authors studied morphological operators on graphs [14,5,313]. However, to the best of our knowledge, very few studies exist about basic morphological operators on complexes [8], and none deal with the filtering problem. The goal of this 
paper is to help bridging this gap. Our main result is a framework for building morphological operators on complex spaces. As main examples of application, we present a set of operators (erosions/dilations, granulometries/anti-granulometry, and alternate sequential filters) that act on the subcomplexes of a space which is itself a simplicial complex. Although this work is settled in the framework of simplicial complexes, all the results extend to cubical complexes.

The article is organized as follows. Section 2 presents the working space, simplicial complexes and lattices. Then, Section 3 introduces operators acting on the defined spaces and shows that these operators are dilations, identifies their adjoint erosions, and presents morphological operators on subcomplexes resulting from the composition of these adjoint operators. Finally, Section 4 introduces a framework that allows morphological operators depending on dimension parameters to be defined and illustrates their use to image and mesh processing.

\section{Lattice of Simplicial Complexes}

The goal of this work is to explore mathematical morphology on simplicial complex spaces. To this end, this first section recalls the definition of (abstract) simplicial complexes. Then, after a reminder on lattices, we state that the set of all subcomplexes of the space is a lattice. Hence, as will show the next sections of the paper, morphological operators acting on subcomplexes can be studied.

We call (abstract) simplex any finite nonempty set. The dimension of a simplex $x$, denoted by $\operatorname{dim}(x)$, is the number of its elements minus one. A simplex of dimension $n$ is also called an $n$-simplex.

Fig. 1(a) (resp. b, and c) graphically represents a simplex $x=\{a\}$ (resp. $y=\{a, b\}$ and $z=\{a, b, c\}$ ) of dimension 0 (resp. 1,2). Fig. 1(d) shows a set of simplices composed of one 2-simplex $(\{a, b, c\})$, three 1-simplices $(\{a, b\},\{b, c\}$ and $\{a, c\})$ and three 0 -simplices $(\{a\},\{b\}$ and $\{c\})$.

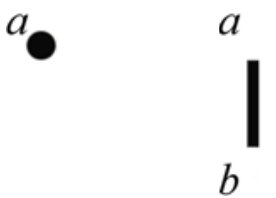

(a) (b)

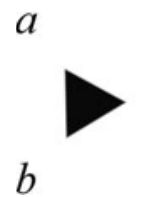

(c)

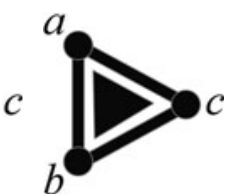

(d)

Fig. 1. Graphical representation of (a) a 0-simplex, (b) a 1-simplex, (c) a 2-simplex, and (d) a 2-cell

We call simplicial complex, or simply complex, any set $X$ of simplices such that, for any $x \in X$, any nonempty subset of $x$ also belongs to $X$. The dimension of a complex is equal to the greatest dimension of its simplices. In the following, a complex of dimension $n$ is also called an $n$-complex.

For instance, Fig. 1(d) represents an elementary 2-complex. The set of black and gray elements in Fig. 2(a) represents a 2-complex made of more simplices. 
Important Notations. In this work, the symbol $\mathbb{C}$ denotes a nonempty $n$ complex, with $n \in \mathbb{N}$. This complex $\mathbb{C}$ stands for our working space in this paper. The set of all subsets of $\mathbb{C}$ is denoted by $\mathcal{P}(\mathbb{C})$.

Recall that a (complete) lattice is a partially ordered set in which any two elements have a unique least upper bound, called supremum, and a unique greatest lower bound, called infimum. Such structures are of particular importance for mathematical morphology. Note that the set $\mathcal{P}(\mathbb{C})$, equipped with the inclusion relation, is a lattice. In this lattice $\mathcal{P}(\mathbb{C})$, the supremum and the infimum are respectively the union and the intersection.

Any subset of $\mathbb{C}$ that is also a complex is called a subcomplex (of $\mathbb{C}$ ). We denote by $\mathcal{C}$ the set of all subcomplexes of $\mathbb{C}$. The set $\mathcal{C}$ equipped with the inclusion relation, is a sublattice of $\mathcal{P}(\mathbb{C})$ since $\mathcal{C}$ is a subset of $\mathcal{P}(\mathbb{C})$ closed under union and intersection.

A subcomplex $X$ of $\mathbb{C}$ is called a cell of $\mathbb{C}$ if there exists a simplex $x$ in $X$ such that $X$ is exactly the set of all subsets of $x$. Any subcomplex $X \in \mathcal{C}$ is supgenerated by the family $\mathcal{G}$ of all cells of $\mathbb{C}$ that are included in $X: X=\cup\{Y \in \mathcal{G}\}$. Conversely, any family $\mathcal{G}$ of cells sup-generates an element of $\mathcal{C}$. In this sense, the cells can be seen as the elementary building blocks of the complexes.

If $X$ is a subset of $\mathbb{C}$, we denote by $\bar{X}$ the complement of $X$ (in $\mathbb{C}$ ): $\bar{X}=\mathbb{C} \backslash X$.

The complement of a subcomplex of $\mathbb{C}$, in general, is not a subcomplex. For instance, observe in Fig. 2(a) that, if $\mathbb{C}$ is the complex represented in black and gray, then the complement of the gray subcomplex $X$ is not a subcomplex. Thus, contrarily to the lattice $\mathcal{P}(\mathbb{C})$ which is complemented, the lattice $\mathcal{C}$ is not.

Any subset $X$ of $\mathbb{C}$ whose complement $\bar{X}$ is a subcomplex is called a star (in $\mathbb{C}$ ). We denote by $\mathcal{S}$ the set of all stars in $\mathbb{C}$. As $\mathcal{C}, \mathcal{S}$ is a sublattice of $\mathcal{P}(\mathbb{C})$.

Note that the intersection $\mathcal{C} \cap \mathcal{S}$ is nonempty since it always contains $\emptyset$ and $\mathbb{C}$.

The lattice $\mathcal{C}$ is our main working space in the rest of the paper. As a conclusion to this section, let us summarize its properties as follows.

Property 1. The set $\mathcal{C}$ of the subcomplexes of $\mathbb{C}$ is a complete lattice supgenerated by the set of all cells of $\mathbb{C}$; this lattice is not complemented.

\section{Morphological Operators on Simplicial Complex Spaces}

Our goal is to investigate morphological dilations and erosions that act on complexes, (where both the inputs and the outputs of the operators are complexes), and that produce nontrivial granulometries, (i.e., granulometries where the dilations are not idempotent). Indeed, such nontrivial granulometries are known to be important in mathematical morphology for analyzing and filtering digital objects according to their size. After a short reminder on morphological adjunctions in the framework of lattices, we present operators that are classical for handling topological spaces such as simplicial complexes. Then, we show that dilations, erosions and granulometries satisfying the above-mentioned properties can be obtained by carefully composing these topological operators.

In mathematical morphology (see, e.g., [12]), any operator that associates elements of a lattice $\mathcal{L}_{1}$ to elements of a lattice $\mathcal{L}_{2}$ is called a dilation if it commutes 
with the supremum. Similarly, an operator that commutes with the infimum is called an erosion. The notion of adjunction, recalled below, allows dilations and erosions to be classified into pairs of operators leading to granulometries.

Let $\mathcal{L}_{1}$ and $\mathcal{L}_{2}$ be two lattices whose order relations and suprema are denoted by $\leq_{1}, \leq_{2}, \vee_{1}$, and $\vee_{2}$. Two operators $\alpha: \mathcal{L}_{2} \rightarrow \mathcal{L}_{1}$ and $\alpha^{A}: \mathcal{L}_{1} \rightarrow \mathcal{L}_{2}$ form an adjunction $\left(\alpha^{A}, \alpha\right)$ if $\alpha(a) \leq_{1} b \Leftrightarrow a \leq_{2} \alpha^{A}(b)$ for every element $a$ in $\mathcal{L}_{2}$ and $b$ in $\mathcal{L}_{1}$. It is well known (see, e.g., 12 ) that, given two operators $\alpha$ and $\alpha^{A}$, if the pair $\left(\alpha^{A}, \alpha\right)$ is an adjunction, then $\alpha^{A}$ is an erosion and $\alpha$ is a dilation. Furthermore, if $\alpha$ is a dilation, the following relation characterizes its adjoint erosion $\alpha^{A}$ :

$$
\forall a \in \mathcal{L}_{1}, \alpha^{A}(a)=\vee_{2}\left\{b \in \mathcal{L}_{2} \mid \alpha(b) \leq_{1} a\right\}
$$

Let us now present two pairs of adjoint operators, which are classical in topology, and that will serve us to obtain nontrivial granulometries on complexes. Let $x$ be a simplex in $\mathbb{C}$, we set $\hat{x}=\{y \mid y \subseteq x, y \neq \emptyset\}$ and $\check{x}=\{y \in \mathbb{C} \mid x \subseteq y\}$.

The operators $C l: \mathcal{P}(\mathbb{C}) \rightarrow \mathcal{P}(\mathbb{C})$ and $S t: \mathcal{P}(\mathbb{C}) \rightarrow \mathcal{P}(\mathbb{C})$ are defined by:

$$
\begin{aligned}
& \forall X \in \mathcal{P}(\mathbb{C}), C l(X)=\bigcup\{\hat{x} \mid x \in X\} ; \text { and } \\
& \forall X \in \mathcal{P}(\mathbb{C}), \operatorname{St}(X)=\bigcup\{\check{x} \mid x \in X\} .
\end{aligned}
$$

By definition, the operators $C l$ and $S t$ commute under union. Thus, they are dilations on $\mathcal{P}(\mathbb{C})$. Hence, by direct application of Eq. (1), the adjoint erosions $C l^{A}$ and $S t^{A}$ of $C l$ and $S t$ are given by:

$$
\begin{aligned}
& \forall X \in \mathcal{P}(\mathbb{C}), C l^{A}(X)=\bigcup\{Y \in \mathcal{P}(\mathbb{C}) \mid C l(Y) \subseteq X\} ; \text { and } \\
& \forall X \in \mathcal{P}(\mathbb{C}), S t^{A}(X)=\bigcup\{Y \in \mathcal{P}(\mathbb{C}) \mid S t(Y) \subseteq X\} .
\end{aligned}
$$

The four operators presented above are illustrated in Fig. 2, where the subsets $X, Y, Z, V$, and $W$, made of gray simplices in Figs. 2(a), 2(b), 2(c), 2(d), and $2(\mathrm{e})$, satisfy the following relations $Y=S t(X), Z=S t^{A}(X), V=C l(Y)$, $W=C l^{A}(Z)$.

Let $X \in \mathcal{P}(\mathbb{C})$. The set $C l(X)$ (resp. $S t(X))$ is the smallest complex (resp. star) that contains $X$, and the set $C l^{A}(X)$ (resp. $\left.S t^{A}(X)\right)$ is the largest complex (resp. star) contained in $X$. Hence, clearly, $\mathcal{C}$ (resp. $\mathcal{S}$ ) is the invariance domain of $C l$ and $C l^{A}$ (resp. St and $\left.S t^{A}\right): \mathcal{C}=\{X \in \mathcal{P}(\mathbb{C}) \mid C l(X)=X\}=$ $\left\{X \in \mathcal{P}(\mathbb{C}) \mid C l^{A}(X)=X\right\}$ (resp. $\mathcal{S}=\{X \in \mathcal{P}(\mathbb{C}) \mid S t(X)=X\}=\{X \in$ $\left.\left.\mathcal{P}(\mathbb{C}) \mid S t^{A}(X)=X\right\}\right)$. These facts are well known in the context of topological spaces [6] where the sets $S t(X), S t(X), C l^{A}(X)$, and $S t^{A}(X)$ are called respectively the (simplicial) closure, the star, the core, and the interior of $X$.

Since the operators $C l$ and $S t$ are dilations, they constitute a straightforward choice to investigate morphology on complexes. However, these dilations are idempotent: $C l \circ C l(X)=C l(X)$ and $S t \circ S t(X)=S t(X)$. Thus, they lead to trivial granulometries. In order to obtain nontrivial granulometries, one could consider the composition $D i l=C l \circ S t$. Indeed, the operator $D i l$ is a dilation (since it is a composition of dilations), which, in general, is not idempotent, and whose results are always complexes. By the theorem of composition of adjunctions (see [12, p. 59), the adjoint erosion Er of Dil is given 
by $E r=D i l^{A}=S t^{A} \circ C l^{A}$. Due to the remarks of the previous paragraph, the set $\operatorname{Er}(X)$ is always a star. Thus, in general, the set $\operatorname{Er}(X)$ is not a complex. Hence, the pair $(E r, D i l)$ does not lead to granulometries acting on complexes.

In order to obtain nontrivial granulometries on complexes, let us restrict the operators of Eqs. 2 and 3 . More precisely, we define the operators $\diamond: \mathcal{S} \rightarrow \mathcal{C}$ and $\star: \mathcal{C} \rightarrow \mathcal{S}$ by:

$$
\begin{aligned}
\forall X \in \mathcal{S}, \diamond(X) & =C l(X) ; \text { and } \\
\forall Y \in \mathcal{C}, \star(Y) & =S t(Y) .
\end{aligned}
$$

The only differences between $\diamond$ and $C l$ are the domains of activity of the operators. A similar remark holds true for $\star$ and St. These operators $\diamond$ and $\star$ are also obviously two dilations. Then, using again Eq. 1, the adjoint erosions $\diamond^{A}$ and $\star^{A}$ of $\diamond$ and $\star$ are given by:

$$
\begin{aligned}
& \forall X \in \mathcal{C}, \diamond^{A}(X)=\bigcup\{Y \in \mathcal{S} \mid \diamond(Y) \subseteq X\} ; \text { and } \\
& \forall Y \in \mathcal{S}, \star^{A}(Y)=\bigcup\{X \in \mathcal{C} \mid \star(X) \subseteq Y\} .
\end{aligned}
$$

It can be easily seen that the star $\diamond^{A}(X)$ is the interior of the complex $X$ and that the complex $\star^{A}(Y)$ is the core of the star $Y$. Therefore, one straightforwardly deduces the following property that links the adjoint of $\star, S t, \diamond$, and $C l$ in a surprising way.

Property 2. The two following propositions hold true:

$$
\begin{aligned}
& \forall X \in \mathcal{C}, \diamond^{A}(X)=S t^{A}(X) ; \text { and } \\
& \forall Y \in \mathcal{S}, \star^{A}(Y)=C l^{A}(Y) .
\end{aligned}
$$

It is known in topology that the closure and interior operators are dual with respect to the complement. Thus, we deduce the following result.

Property 3. The operators $\diamond$ and $\diamond^{A}$ (resp. $\star$ and $\left.\star^{A}\right)$ are dual w.r.t. the complement in $\mathcal{P}(\mathbb{C})$ : we have $\diamond^{A}(X)=\overline{\diamond(\bar{X})}$, for any $X \in \mathcal{C}\left(\right.$ resp. $\star^{A}(Y)=\overline{\star(\bar{Y})}$, for any $Y \in \mathcal{S})$

Note that using directly Eqs. 8 and 9 computing $\diamond^{A}(X)\left(\right.$ resp. $\left.\star^{A}(X)\right)$ requires an exponential time since the family of all stars (complexes) must be considered. On the other hand, as the operators $C l$ and $S t$ are locally defined, $\diamond(X)$ and $\star(X)$ can be computed in linear-time. Hence, as a consequence of Property 3 , $\diamond^{A}(X)$ and $\star^{A}(X)$ can also be computed in linear-time.

Let us now compose the dilations $\diamond$ and $\star$, as well as their adjoints, to obtain a pair of adjoint dilations and erosions that act on complexes.

Definition 4. We define the operators $\delta$ and $\varepsilon$ acting on $\mathcal{C}$ by:

$$
\begin{aligned}
& \delta=\diamond \circ \star \\
& \varepsilon=\star^{A} \circ \diamond^{A}
\end{aligned}
$$




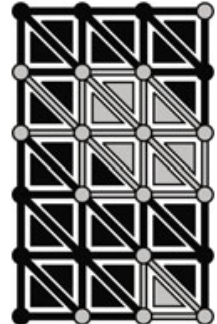

(a) $X$

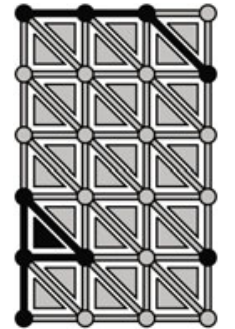

(b) $Y$

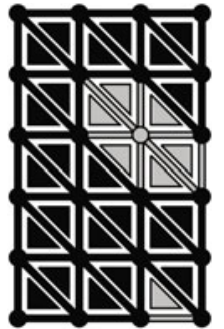

(c) $Z$

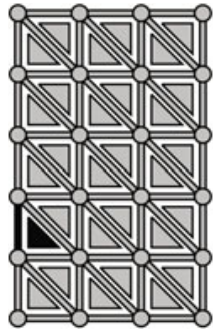

(d) $\mathrm{V}$

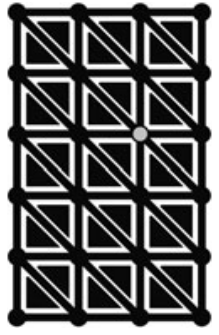

(e) $W$

Fig. 2. Illustration of morphological dilations and erosions on complexes [see text]

For instance, Figs. 2(d) and 2(e) represent, in gray, the complexes $V=\delta(X)$ and $W=\varepsilon(X)$, if $X$ is the complex represented in gray in Fig. 2(a).

Due to the theorem of composition of adjunctions (see, e.g., [12], p. 59), the following result can be deduced.

Theorem 5. The two operators $\delta$ and $\varepsilon$ are respectively a dilation and an erosion acting on $\mathcal{C}$. Furthermore, the pair $(\varepsilon, \delta)$ is an adjunction.

The dilation $\delta$ and the erosion $\varepsilon$, as well as the classical dilations and erosions on grid points by symmetrical structuring elements, are in general not idempotent. However, contrarily to classical dilations and erosions on grid points, the erosion $\varepsilon$ and the dilation $\delta$ are not dual with respect to the complement since the lattice $\mathcal{C}$ is not complemented.

As the pair $(\varepsilon, \delta)$ is an adjunction, the compositions $\phi=\varepsilon \circ \delta$ and $\gamma=$ $\delta \circ \varepsilon$ are respectively a closing and an opening. In other words, the operators $\phi$ and $\gamma$ are both increasing and idempotent, whereas $\phi$ is extensive and $\gamma$ is antiextensive. Furthermore, since $\delta$ and $\varepsilon$ are not idempotent, the operators obtained by iterating $\varepsilon$ and $\delta$ lead to nontrivial granulometries, as we will detail in the next section.

Figs. 3(b) and 3(d) depict, in gray, the results of $\phi$ and $\gamma$ applied to the gray complexes $X$ of Fig. 3(a) and $Y$ of Fig. 3(c). Observe, in particular, that these operators can be intuitively regarded as elementary closing and opening on complexes.

\section{Dimensional Operators}

From their very definition, simplicial complex spaces allow for handling objects of different dimension (e.g., "curvilinear", "surfacic" or "volumic" objects), as well as objects of heterogeneous dimension (e.g., made of "curvilinear", "surfacic" and "volumic" sub-objects). The operators introduced in the previous section add or remove simplices independently of the dimension of the objects. In this section, we introduce a framework for morphological operators that take dimension into account. We first present (Definition 6) the building blocks of the 


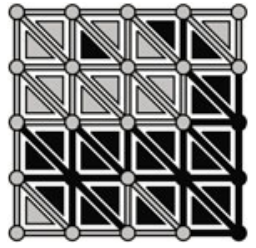

(a) $X$

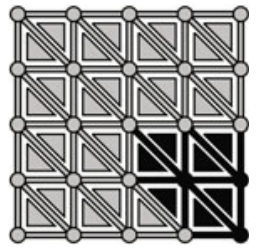

(b) $\phi(X)$

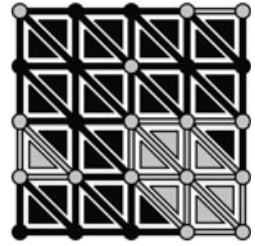

(c) $Y$

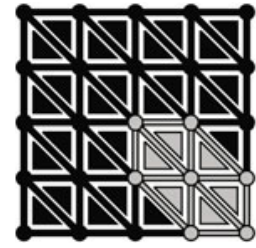

(d) $\gamma(Y)$

Fig. 3. Illustration of morphological closing and opening on complexes

framework: a family of adjoint operators that depend on dimensional parameters. By combining these building blocks, many morphological operators acting on subcomplexes, substars, and subsets of the space $\mathbb{C}$ can be obtained. As an example, we instantiate (Definition 9) a granulometry and an alternate sequential filter acting on the lattice of all subcomplexes of $\mathbb{C}$. Then, it is shown that these filters lead to interesting results on images, as suggested by a comparison with classical morphological image filters. Finally, the ability of the proposed filters to smooth subsets of a mesh is also illustrated.

Let $X \subseteq \mathbb{C}$ and let $i \in[0, n]$, we denote by $X_{i}$ the set of all $i$-simplices of $X: X_{i}=\{x \in X \mid \operatorname{dim}(x)=i\}$. In particular, $\mathbb{C}_{i}$ is the set of all $i$-simplices of $\mathbb{C}$. We denote by $\mathcal{P}\left(\mathbb{C}_{i}\right)$ the set of all subsets of $\mathbb{C}_{i}$.

Definition 6 (dimensional operators). Let $i, j \in \mathbb{N}$ such that $i \leq j \leq n$. We define the operators $\delta_{i, j}^{+}$and $\varepsilon_{i, j}^{+}$acting from $\mathcal{P}\left(\mathbb{C}_{i}\right)$ into $\mathcal{P}\left(\mathbb{C}_{j}\right)$ and the operators $\delta_{j, i}^{-}$and $\varepsilon_{j, i}^{-}$acting from $\mathcal{P}\left(\mathbb{C}_{j}\right)$ into $\mathcal{P}\left(\mathbb{C}_{i}\right)$ as follows:

\begin{tabular}{|l|l|}
\hline $\mathcal{P}\left(\mathbb{C}_{i}\right) \rightarrow \mathcal{P}\left(\mathbb{C}_{j}\right)$ & $\mathcal{P}\left(\mathbb{C}_{j}\right) \rightarrow \mathcal{P}\left(\mathbb{C}_{i}\right)$ \\
\hline$X \rightarrow \delta_{i, j}^{+}(X)$ such that & $X \rightarrow \delta_{j, i}^{-}(X)$ such that \\
$\delta_{i, j}^{+}(X)=\left\{x \in \mathbb{C}_{j} \mid \exists y \in X, y \subseteq x\right\}$ & $\delta_{j, i}^{-}(X)=\left\{x \in \mathbb{C}_{i} \mid \exists y \in X, x \subseteq y\right\}$ \\
\hline$X \longrightarrow \varepsilon_{i, j}^{+}(X)$ such that & $\begin{array}{l}X \longrightarrow \varepsilon_{j, i}^{-}(X) \text { such that } \\
\varepsilon_{i, j}^{+}(X)=\left\{x \in \mathbb{C}_{j} \mid \forall y \in \mathbb{C}_{i}, y \subseteq\right. \\
x \Longrightarrow y \in X\}\end{array}$ \\
$\begin{array}{l}\varepsilon_{j, i}^{-}(X)=\left\{x \in \mathbb{C}_{i} \mid \forall y \in \mathbb{C}_{j}, x \subseteq\right. \\
y \Longrightarrow y \in X\}\end{array}$ \\
\hline
\end{tabular}

In other words, $\delta_{i, j}^{+}(X)$ is the set of all $j$-simplices of $\mathbb{C}$ that include an $i$-simplex of $X, \delta_{j, i}^{-}(X)$ is the set of all $i$-simplices of $\mathbb{C}$ that are included in a $j$-simplex of $X, \varepsilon_{i, j}^{+}(X)$ is the set of all $j$-simplices of $\mathbb{C}$ whose subsets of dimension $i$ all belong to $X$, and $\varepsilon_{j, i}^{-}(X)$ is the set of all $i$-simplices of $\mathbb{C}$ that are not contained in any $j$-simplex of $\bar{X}$.

It is interesting to remark that, since a graph is a 1-complex, the operators $\delta_{0,1}^{+}, \delta_{1,0}^{-}, \varepsilon_{0,1}^{+}$and $\varepsilon_{1,0}^{-}$are exactly the operators $\delta^{\times}, \delta^{\bullet}, \varepsilon^{\times}$and $\varepsilon^{\bullet}$, presented by Cousty et al. in [3]. These operators are the basic blocks of all morphological graph operators studied in [3]. Thus, the present framework encompasses the one of [3] that itself allows for recovering most graph operators from Vincent and Heijmans [145, the operators of Meyer and Angulo [9], and the classical operators by symmetric structuring elements on grid points. 
The next property establishes that the dimensional operators of Definition 6 can be classified into pairs of adjoint operators. Thus, as we shall see later, they can be used as building blocks for the design of morphological operators acting on subsets of $\mathbb{C}$ such as complexes or stars.

Property 7 (adjunction and duality). Let $i, j \in \mathbb{N}$ such that $i \leq j \leq n$.

- The pairs $\left(\varepsilon_{i, j}^{+}, \delta_{j, i}^{-}\right)$and $\left(\varepsilon_{j, i}^{-}, \delta_{i, j}^{+}\right)$are adjunctions.

- The operators $\delta_{i, j}^{+}$and $\varepsilon_{i, j}^{+}$(resp. $\delta_{j, i}^{-}$and $\varepsilon_{j, i}^{-}$) are dual of each other: $\forall X \subseteq \mathbb{C}_{i}$, $\varepsilon_{i, j}^{+}(X)=\mathbb{C}_{j} \backslash \delta_{i, j}^{+}\left(\mathbb{C}_{i} \backslash X\right)$ (resp. $\left.\forall X \subseteq \mathbb{C}_{j}, \varepsilon_{j, i}^{-}(X)=\mathbb{C}_{i} \backslash \delta_{j, i}^{-}\left(\mathbb{C}_{j} \backslash X\right)\right)$.

The operators of Section 3 can all be characterized through dimensional operators since, for any $X$ in $\mathcal{P}(\mathbb{C})$, we have $C l(X)=\bigcup\left\{\delta_{j, i}^{-}\left(X_{j}\right) \mid i, j \in \mathbb{N}, i \leq j\right\}$, and $S t(X)=\bigcup\left\{\delta_{i, j}^{+}\left(X_{i}\right) \mid i, j \in \mathbb{N}, i \leq j\right\}$.

In fact, by compositions, suprema, and infima of the dimensional operators, many erosions, dilations, openings and closings, which act on $\mathcal{C}, \mathcal{S}, \mathcal{P}\left(\mathbb{C}_{i}\right)$ (with $i \in[0, n]$ ), and $\mathcal{P}(\mathbb{C})$, and which depend of dimensional parameters, can be designed. As a proof of concept, let us introduce a family of filters that act on the lattice of all subcomplexes of $\mathbb{C}$ and that enriches the granulometries obtained from Section 3 .

Definition 8. Let $d$ be an integer such that $0 \leq d \leq n$ and let $X \in \mathcal{C}$. We define the operators $\gamma_{d /(n+1)}$ and $\phi_{d /(n+1)}$ by, for any $X \in \mathcal{C}$.

$$
\begin{gathered}
\gamma_{d /(n+1)}(X)=\bigcup\left\{\delta_{j, i}^{-}\left(X_{j}\right) \mid j \in[d, n], i \in[0, j]\right\} ; \text { and } \\
\phi_{d /(n+1)}(X)=\bigcup\left[\left\{X_{i} \mid i \in[0, n-d-1]\right\} \cup\left\{\varepsilon_{n-d, j}^{+}\left(X_{d}\right) \mid j \in[n-d, n]\right\}\right]
\end{gathered}
$$

For instance Figs. 4(a), 4(b) 4(c) and 4(d) represent in gray the complexes $\phi_{1 / 3}(X), \phi_{2 / 3}(X), \gamma_{1 / 3}(Y)$, and $\gamma_{2 / 3}(Y)$, where $X$ and $Y$ are the subcomplexes shown in gray in Figs. $3(\mathrm{a})$ and $3(\mathrm{c})$.

Let $d \in[0, n]$, and let $X$ be a subcomplex of $\mathbb{C}$. It can be seen that $\gamma_{d /(n+1)}(X)$ is the union of the cells of dimension greater than or equal to $d$ that are included in $X$. On the other hand, $\phi_{d /(n+1)}(X)$ is the union of the cells of $X$ and of those of $\mathbb{C}$ whose elements of dimension between 0 and $n-d$ belong to $X$.

From these characterizations, we can deduce that the operators $\gamma_{d /(n+1)}$ and $\phi_{d /(n+1)}$ are respectively an opening and a closing on $\mathcal{C}$.

It can also be remarked that the family of operators $\left\{\gamma_{d /(n+1)} \mid d \in[0, n]\right\}$ and $\left\{\phi_{d /(n+1)} \mid d \in[0, n]\right\}$ are ordered with respect to the value of the in$\operatorname{dex} d: \forall X \in \mathcal{C}, \forall d_{1}, d_{2} \in[0, n-1]$, if $d_{1} \leq d_{2}$, then we have $\phi_{d_{1} /(n+1)}(X) \subseteq$ $\phi_{d_{2} /(n+1)}(X)$ and $\gamma_{d_{2} /(n+1)}(X) \subseteq \gamma_{d_{1} /(n+1)}(X)$. In morphology, such families are called granulometries. For direct applications to real-life problems, these granulometries may appear useless since they contain only a fixed number $n$ of operators whereas the size of the objects to be analyzed can be arbitrarily large. In order to overcome this problem, in the next definition, these granulometries are extended by composing them with the adjunctions (and their iterated version) of Section 3 . 


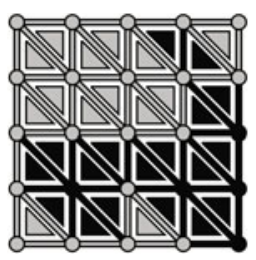

(a) $\phi_{1 / 3}(X)$

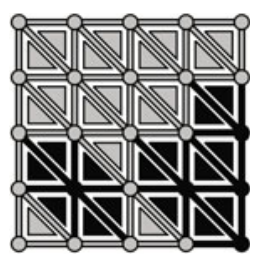

(b) $\phi_{2 / 3}(X)$

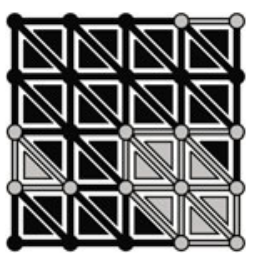

(c) $\gamma_{1 / 3}(Y)$

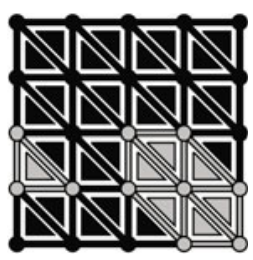

(d) $\gamma_{2 / 3}(Y)$

Fig. 4. Dimensional openings and closings applied to the complexes $X$ and $Y$ of Fig. 3

Let $\alpha$ be an operator defined on a lattice $\mathcal{L}$ and let $i$ be a nonnegative integer. The operator $\alpha^{i}$ is defined by the identity when $i=0$ and by $\alpha \circ \alpha^{i-1}$ otherwise.

Definition 9 (granulometry, ASF). Let $k$ be a nonnegative integer.

1. We define $\Gamma_{k /(n+1)}\left(\right.$ resp. $\left.\Phi_{k /(n+1)}\right)$ by $\delta^{i} \circ \gamma_{d /(n+1)} \circ \varepsilon^{i}\left(\right.$ resp. $\varepsilon^{i} \circ \phi_{d /(n+1)} \circ$ $\left.\delta^{i}\right)$ where $i$ and $d$ denote respectively the quotient and the remainder of the integer division of $k$ by $(n+1)$

2. We define $A S F_{k /(n+1)}$ by the identity when $k=0$ and by $A S F_{k /(n+1)}=$ $\Gamma_{k / n+1} \circ \Phi_{k / n+1} \circ A S F_{(k-1) /(n+1)}$ otherwise.

Property 10. 1. The families $\left\{\Gamma_{k /(n+1)} \mid k \in \mathbb{N}\right\}$ and $\left\{\Phi_{k /(n+1)} \mid k \in \mathbb{N}\right\}$ are granulometries:

- for any $k \in \mathbb{N}, \Gamma_{k /(n+1)}$ (resp. $\Phi_{k /(n+1)}$ ) is an opening (resp. closing) on $\mathcal{C}$.

- for any two elements $i, j \in \mathbb{N}$ such that $i \leq j$, we have $\Gamma_{j /(n+1)}(X) \subseteq$ $\Gamma_{i /(n+1)}(X)$ and $\Phi_{i /(n+1)}(X) \subseteq \Phi_{j /(n+1)}(X)$, for any $X \in \mathcal{C}$.

2. The family $\left\{A S F_{k /(n+1)} \mid k \in \mathbb{N}\right\}$ is a family of alternate sequential filters:

- for any two elements $i, j \in \mathbb{N}$, if $i \leq j$, then we have $A S F_{j /(n+1)} \circ$ $A S F_{i /(n+1)}=A S F_{j /(n+1)}$.

Note that if we reduce the granulometric and ASF families of Definition 9] to the operators where $k$ is a multiple of $(n+1)$, then we recover exactly the granulometries and ASFs induced by the adjunctions of Section 3. In this sense, the proposed granulometries and ASFs enrich the elementary ones on complexes.

Let us now illustrate on an 2D image that, in practice, these operators also enrich the classical morphological filters used in image analysis. To this end , we consider simplicial complexes based on the neighborhood relation given by the hexagonal grid (see e.g., Fig. 2(a) where the grid points (or pixels) correspond to 0 -simplices. In this context, Fig. $5(\mathrm{f})$ shows the result of $A S F_{6 / 3}$ applied to the complex derived from the white pixels of image $5(\mathrm{a})$. For comparison, Fig. 5(c) and Fig. 5(e) show the results of classical alternate sequential filter and graph alternate sequential filters [3] on hexagonal grid. On this image, the ASFs introduced in this paper outperform the classical and graph operators. Nevertheless, they require more iterations (6 vs. 2 and 4). Therefore, in order to compare the proposed ASF with filters using the same number of iterations, 


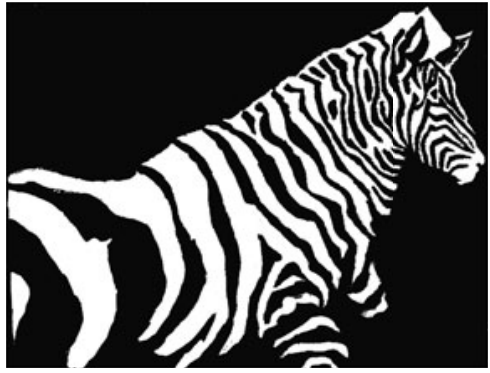

(a) Original image.

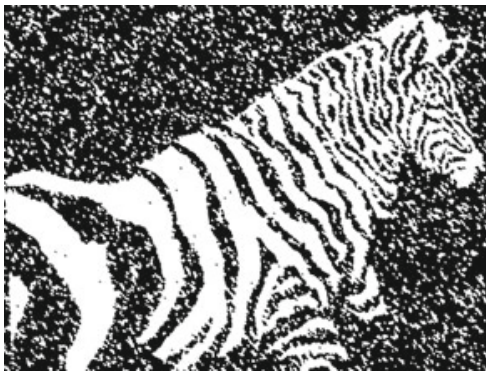

(c) Classical ASF with 2 iterations. $M S E=16.14 \%$.

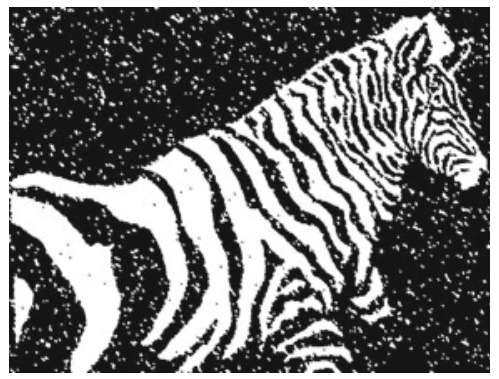

(e) Graph $A S F_{4 / 2}$ 3] (see text). $M S E=6.88 \%$.

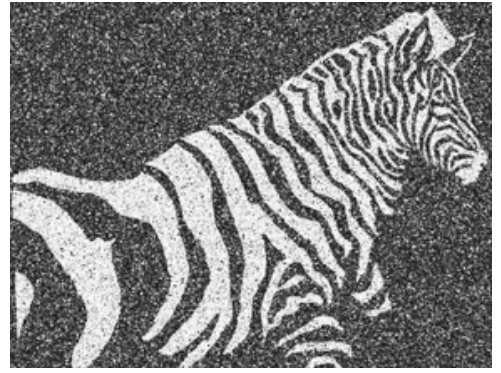

(b) Noisy version, $M S E=19.56 \%$.

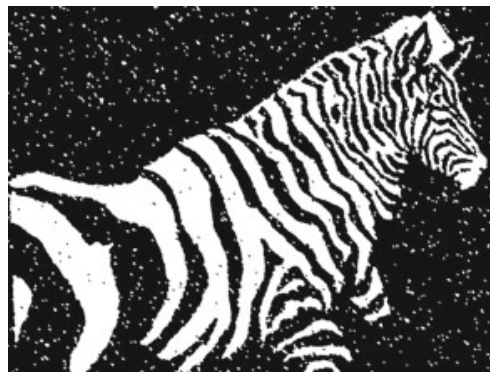

(d) Classical ASF with 6 iterations and triple resolution. $M S E=$ $4.05 \%$.

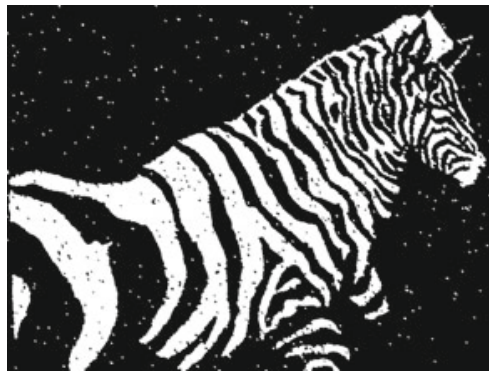

(f) $A S F_{6 / 3} \cdot M S E=2.57 \%$.

Fig. 5. ASF illustration [see text]

we produce another filtered image (Fig. $5(\mathrm{~d})$ that is obtained by tripling the resolution of the noisy image and applying a classical ASF of size $2 \times 3=6$. It can be seen that this last procedure removes more noise than the classical ASF but does not perform as well as the ASF introduced in the present paper. The mentioned value $M S E$ is the mean square error, multiplied by 100 , that is, the percentage of wrong pixels w.r.t. the original image.

Fig. 6(a) shows a rendering of a tridimensional mesh of a statue. Fig. 6(b) shows, in black, the complex resulting from the threshold at level 0.51 of the pseudo-inverse of the mean curvature of that mesh (see [10] for further details 


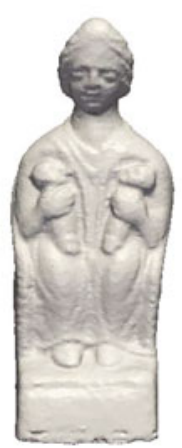

(a)

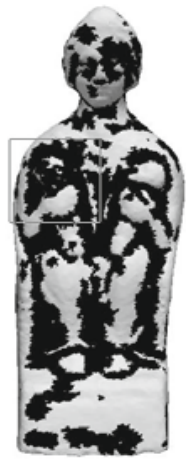

(b)

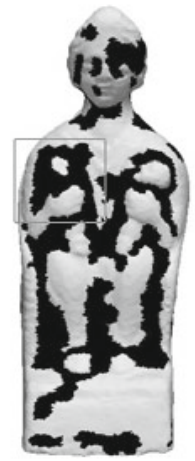

(c)

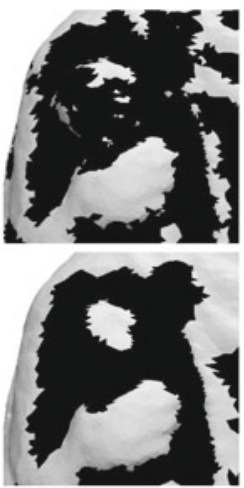

(d)

Fig. 6. (a) Rendering of a tridimensional mesh (i.e. a 2-complex embedded in $\mathbb{R}^{3}$ ). (b) a threshold of the curvature map of (a). (c) Result of the operator $A S F_{8 / 3}$ applied to the subcomplex shown in black in (b). (d) Crops, from top to bottom, of (b) and (c) respectively. Data courtesy of the French Museum Center for Research.

and motivations on such procedures). To illustrate the possible smoothing effect of the operator on values associated to a mesh, we present in Fig. 6(c) the result of $A S F_{8 / 3}$ applied to the black subset of the mesh shown on Fig. 6(b) Fig. 6(d) presents, from top to bottom, zooms on the parts of Fig. 6(b) and 6(c) that are marked by bold rectangles.

\section{Conclusion and Future Work}

This paper proposes a framework that allows to build morphological operators for analyzing and filtering objects defined on simplicial complex spaces. In particular, using this framework, we propose a set of operators (erosions/dilations, granulometries, and alternate sequential filters), which act on the lattice of subcomplexes, that are shown to be useful for mesh and image filtering. Furthermore, the proposed framework extends straightforwardly to define operators with similar behavior acting on the lattice of stars of $\mathbb{C}$. Future work includes a systematic investigation of the morphological operators that can be built based on our framework as well as its straightforward extension to weighted simplicial complexes $4 / 2$. In particular, links with operators from discrete calculus will be highlighted.

Acknowledgement. The authors are grateful to Christian Ronse for his indepth reading of a previous version of this paper and his numerous helpful comments. 


\section{References}

1. Couprie, M., Bertrand, G.: New characterizations of simple points in 2D, 3D and 4D discrete spaces. IEEE Trans. Pattern Analysis and Machine Intelligence 31(4), 637-648 (2009)

2. Cousty, J., Bertrand, G., Couprie, M., Najman, L.: Collapses and watersheds in pseudomanifolds. In: Wiederhold, P., Barneva, R.P. (eds.) IWCIA 2009. LNCS, vol. 5852, pp. 397-410. Springer, Heidelberg (2009)

3. Cousty, J., Najman, L., Serra, J.: Some morphological operators in graph spaces. In: Wilkinson, M.H.F., Roerdink, J.B.T.M. (eds.) ISMM 2009. LNCS, vol. 5720, pp. 149-160. Springer, Heidelberg (2009)

4. Grady, L.J., Polimeni, J.R.: Discrete Calculus. Springer, Heidelberg (2010)

5. Heijmans, H., Vincent, L.: Graph morphology in image analysis. In: Dougherty, E. (ed.) Mathematical Morphology in Image Processing, pp. 171-203. Marcel Dekker, New York (1992)

6. Jänich, K.: Topology. Springer, Heidelberg (1984)

7. Kovalevsky, V.A.: Finite topology as applied to image analysis. CVGIP 46(2), 141-161 (1989)

8. Loménie, N., Stamon, G.: Morphological mesh filtering and $\alpha$-objects. Pattern Recognition Letters 29(10), 1571-1579 (2008)

9. Meyer, F., Angulo, J.: Micro-viscous morphological operators. In: ISMM 2007, pp. 165-176 (2007)

10. Philipp-Foliguet, S., Jordan, M., Najman, L., Cousty, J.: Artwork 3D Model Database Indexing and Classification. PR 44(3), 588-597 (2011)

11. Poincaré, H.: Analysis situs. Journal de l'École Polytechniquee 2(1), 1-178 (1895)

12. Ronse, C., Serra, J.: Algebric foundations of morphology. In: Najman, L., Talbot, H. (eds.) Mathematical Morphology: From Theory to Applications, pp. 35-79. ISTE-Wiley, Chichester (2010)

13. Ta, V.T., Elmoataz, A., Lezoray, O.: Partial difference equations on graphs for mathematical morphology operators over images and manifolds. In: Procs. of ICIP 2008, pp. 801-804 (12-15, 2008)

14. Vincent, L.: Graphs and mathematical morphology. Signal Processing 16, 365-388 (1989) 